\title{
Praktiken sozialer Reproduktion von prekär beschäftigten Männern
}

Die Frage, in welcher Weise sich mit der Prekarisierung von Arbeit und Leben die soziale Integration unterschiedlicher Milieus und Gruppen verändert, wird gegenwärtig kontrovers diskutiert. Erst ansatzweise erforscht ist, wie Prekarität von den Einzelnen erlebt und gelebt wird, welche verschiedenen Praktiken sozialer Reproduktion unter den Bedingungen der Prekarisierung entstehen. Soziale Reproduktion meint hier unterschiedliche Strategien sozialen Handelns, die aber durchaus miteinander korrespondieren: das Bemühen der Einzelnen um eine respektable soziale Position und die Praktiken der Selbstsorge und Sorge für andere im Sinne der Regeneration und des Erhalts der eigenen Lebenskraft.

\section{Einleitung}

\subsection{PROBLEMAUFRISS}

Ausgangspunkt des Beitrags ist die in der Arbeits- und Geschlechtersoziologie geführte Debatte darüber, welche Konsequenzen gegenwärtige Prekarisierungsprozesse für die gelebten Geschlechterarrangements und die institutionelle Regulierung des Geschlechterverhältnisses haben. Mit Prekarisierung sind dabei vor allem Dynamiken sozialer Entsicherung gemeint, die sowohl die institutionelle Aufkündigung und Erosion von gesellschaftlichen Vereinbarungen und Interessenausgleichen (zum Beispiel sozialstaatlicher oder arbeitsrechtlicher Absicherungen) als auch den subjektiv als Verunsicherung erlebten Verlust der Deutbarkeit des Laufs der Welt und der Antizipierbarkeit von $\mathrm{Zu}$ kunft betreffen. In der ungleichheits- und arbeitssoziologischen Diskussion werden vor allem drei Phänomene akzentuiert: die Veränderungen in der Organisation von (Erwerbs-)Arbeit (Castel/Dörre 2009) und damit verbundener Geschlechterregime (Manske/Pühl 2010), die neu aufgeworfene soziale Frage nach gesellschaftlicher Integration und nach Kohäsion (Bude/ Willisch 2008; Castel 2000; Götz/Lemberger 2009) und die veränderte Herrschaftslogik und qualitativ neue Form der Subjektivierung (Bourdieu 1998). Anstöße für diese transformatorischen Dynamiken werden oftmals durch Umbrüche und Verwerfungen in der Erwerbsarbeit gegeben, deren Erscheinungsformen von der massiven Ausweitung des Niedriglohnbereichs über die Befristung von Beschäftigung und den Ausbau der Leiharbeitsverhältnisse bis hin zur Expansion geringfügiger Beschäftigung reichen. Die Auswirkungen dieser Umbrüche betreffen ganz unterschiedliche Gruppen; es zeigen sich vielfältige, mehrdimensionale Benachteiligungskonstellationen. Quer dazu gibt es auch Anzeichen für Restrukturierungen vergeschlechtlichter Ungleichheiten zuungunsten von Frauen (Wagner/Wiethold 2009). All diesen komplexen Phänomenen zum Trotz wird die Krise der Arbeit häufig als Krise der Männer betrachtet (Dörre 2007; differenzierend: Lengersdorf/Meuser 2010; Scholz 2009). Es werden dabei vor allem zwei Gesichtspunkte hervorgehoben:

(1) Die sehr ausstrahlungskräftige normative Verknüpfung des Normalarbeitsverhältnisses fordistischer Prägung mit ihrer komplementär-hierarchischen Geschlechterkonstellation (Young 1998) gerät in den Strudel der Entsicherungsprozesse. Sozial anerkannte Männlichkeitsversionen erodieren, wenn die ihnen zugrunde liegenden Erwerbs- und Familienkonstellationen des ernährenden männlichen Haushaltsvorstandes und der weiblichen „Familienerhalterin“ (Krüger 1995) brüchig werden. ${ }^{1}$

(2) Dies ruft vielfach auch persönliche Krisenerfahrungen hervor, die für Angehörige der männlichen Genusgruppe eben stärker mit der Infragestellung ihrer Geschlechtsidentität (und entsprechenden Restabilisierungsaufwänden) verknüpft sein können.

Folgt man der Männlichkeitsforschung, etwa Michael Meuser oder Raewyn Connell, so ist der Strukturwandel der immer stärker transnational organisierten Erwerbsarbeit mit einer Neurelationierung unter- schiedlicher Männlichkeiten verbunden: Im sozialen Raum noch nationalstaatlich organisierter Gesellschaften stehe nun als hegemoniale Konstellation die Elite der „transnational business masculinity“ (vgl. Wedgwood/Connell 2008) ganz oben und präge die Norm der innovativen Arbeitskraft. Dagegen seien die Bewältigungsstrategien der lokal und national gebundenen, zu erheblichen Teilen Ex-Normalarbeitnehmer durch das Festhalten an traditionellen Mustern (Lengersdorf/Meuser 2010) geprägt. Die erhöhte soziale Verwundbarkeit in den unteren oder mittleren sozialen Milieus dieser dominierten Männlichkeiten artikuliere sich überwiegend als Retraditionalisierung ihrer Geschlechterkonstrukte. Damit sind das Errichten von unterschiedenen, voneinander getrennten Männer- und Frauenwelten und das Insistieren auf geschlechtsdifferenzierende Arbeitsteilungen gemeint, die durchaus zur Wahrung des eigenen Terrains dienen können. Andere Hinweise gibt die empirische Studie von Egert et al. (2010), die das Aufbrechen von mehr oder weniger konsistenten Männlichkeiten durch die Nichtübereinstimmung von sozialen Erwartun-

1 Zwar dominierte im hier untersuchten Osten Deutschlands seit der DDR-Frauen- und Familienpolitik das Doppelversorgermodell zweier Vollzeitbeschäftigungsverhältnisse. Dennoch blieben die Geschlechternormen und die Vorstellungen zur Arbeitsteilung dem vergeschlechtlichten Fordismus verhaftet.

Susanne Völker, Prof. Dr., lehrt Soziologie an der Universität zu Köln. Arbeitsschwerpunkte: Geschlechterforschung, gesellschaftliche Transformationsprozesse, Arbeitssoziologie (insb. Prekarisierungsforschung), Methoden der qualitativen Sozialforschung. e-mail: susanne.voelker@uni-koeln.de 
gen und praktischen Lebensführungen als „Praktiken der Nichtmännlichkeit“ bezeichnen und zu dem Schluss kommen, dass Männlichkeit als Kategorie sozialer Verortung mehr und mehr entleert wird. Befunde der Studien von Christina Klenner und Ute Klammer (2010) zu der steigenden Zahl von Familienernährerinnen belegen zudem bescheidene, allerdings nicht durchgängige Neu- und Umverteilungen reproduktiver Tätigkeiten und häuslicher Arbeiten, die teils auf eine pragmatische, teils auf eine bewusst gewählte Rollenverschiebung zurückgehen (vgl. Klenner et al. in diesem Heft).

\subsection{FRAGESTELLUNG UND VORGEHENSWEISE}

Ich möchte die Frage aufgreifen, inwieweit das mehr oder weniger erzwungene Eingehen von prekären Beschäftigungsverhältnissen Männer in Konflikt mit der von ihnen anvisierten sozialen Rolle und/ oder Geschlechtsidentität bringt. Anhand der Auswertung von Interviews mit männlichen Befragten aus mittleren und unteren sozialen Milieus skizziere ich, mittels welcher Selbstdeutungen Männer an fordistisch geprägte Geschlechtervorstellungen anknüpfen und diese unter gesellschaftlich veränderten Bedingungen fortzuführen oder zu aktualisieren versuchen. Zugleich zeige ich, dass sich in den Bezügen auf prekarisierte Beschäftigungsverhältnisse ebenso Tendenzen zur Relativierung der Geschlechterdifferenz und der Bedeutung von Männlichkeit finden lassen. Auffällig ist jedoch, dass die Selbstsorge und Sorge für andere, die physische Regeneration und Reproduktion durch die Prekarisierung von Arbeit und Leben in eine Krise gerät. Kernthese der folgenden Analyse ist daher, dass Geschlecht zwar als Klassifikationsschema zur Deutung von eingegangenen Erwerbsarbeitsverhältnissen für einen Teil von Männern an Bedeutung verliert. Zugleich aber wird Geschlecht in Bezug auf die eigene Reproduktion, im Hinblick auf die Vorstellungen von Familie und einem Leben in sozialen Bindungen stärker relevant gemacht - nicht zuletzt deshalb, weil die Lebenszusammenhänge von den Effizienz- und Verausgabungserfordernissen der Erwerbsarbeit dominiert und bedrängt sind (Aulenbacher 2009). Mitunter scheint für junge Männer ein gelungener und erfüllender Lebenszusammenhang unter prekarisierten Bedingungen unerreichbar oder nur mit Partnervorstellungen umsetzbar, die einen dem Gesellschaftlichen enthobenen Weiblichkeitsmythos bemühen, für den sie im Realen keine Adressatinnen finden. Dies ist m.E. aber nicht hinreichend mit der oben genannten These der Retraditionalisierung der Geschlechtervorstellungen vor allem in den mittleren und unteren Regionen des sozialen Raums zu erfassen, vielmehr zeichnen sich widersprüchliche Prozesse der gleichzeitigen, je nach gesellschaftlichem Teilbereich differenzierten, zu- und abnehmenden Relevanz von Geschlecht ab.

Im Folgenden werde ich anhand von bislang nicht veröffentlichtem qualitativen Interviewmaterial aus einer explorativen Studie zu Beschäftigungsverhältnissen, Lebensführungen und Geschlechterarrangements im ostdeutschen Einzelhan$\mathrm{del}^{2}$ drei Varianten alltagspraktischer Strategien zeigen, mit denen männliche Einzelhandelsbeschäftigte den gewachsenen Instabilitäten ihrer prekarisierten Erwerbs- und Lebensarrangements begegnen (Abschnitte 3-5). Anschließend werden erste Rückschlüsse auf Verschiebungen in den Geschlechterarrangements und die sich zuspitzende Krise der Reproduktion gezogen (Abschnitt 6). Zunächst möchte ich jedoch auf den methodischen Zugang eingehen, durch den überhaupt erst sichtbar wird, welche veränderten, sozial neu kontextualisierten und soziologisch eher vernachlässigten Praktiken hervorgebracht werden (Abschnitt 2).

\section{Zugänge zu Praktiken der Instabilität}

Das Herangehen an das Material, also an die jeweiligen Positionierungen und situativen Deutungen der Interviewten, ist durch zwei methodologische Überlegungen gekennzeichnet:

(1) Es geht hierbei nicht um die Herausarbeitung von aggregierten Typen als verallgemeinerbaren abstrakten Handlungslogiken. Vielmehr wird durch die detaillierte Rekonstruktion von Einzelfällen versucht, das Bewältigen und Hervorbringen von prekarisierten Lebenskonstellationen in ihren komplexen und spezifischen - und das meint auch ihren partikularen - Verknüpfungen zwischen gemachten Erfah- rungen, spontanen Alltagsbewältigungen und dem Umgehen mit Irritationen und Revisionen sichtbar zu machen. Denn - um es in Anknüpfung an Pierre Bourdieu zu formulieren - nicht nur der sozialstrukturelle Verlauf und die Effekte der sozialen Umbrüche sind gegenwärtig ungewiss, auch die Praktiken, mittels derer die Menschen ihnen begegnen. Mit diesen nicht eindeutig antizipierbaren, transformatorischen Dynamiken werden aber zugleich die gewohnten soziologischen Kategorien ungewisser. Sie müssen ihre Triftigkeit erst beweisen, um zu verhindern, dass sozial Neues mit etablierten, beispielsweise mit dem Vorstellungsrahmen fordistischer Gesellschaftskonstellationen aufgeladenen Begriffen, eher unkenntlich wird. Es bedarf daher einer gewissen methodischen Vorsicht, was die Klassifizierung und Typologisierung von sozialem Handeln betrifft, ohne soziologisches Wissen dabei $\mathrm{zu}$ verwerfen, sondern es gerade in seiner Situiertheit zu begreifen. So wäre etwa das in soziologischen Argumentationen verbreitete Insistieren auf einer methodischplanenden Lebensführung als notwendiger Bedingung für ein würdiges Leben als eine begrenzte, historisch spezifische Sichtweise zu behandeln, die durchaus mit den aktuellen Umbrüchen an Geltung verlieren und eben nicht alle Spielarten sozialer Einbindungen erklären kann. Genau hierauf habe ich in dem empirischen Material deutliche Hinweise gefunden.

2 Es handelt sich dabei um die an der Universität Potsdam in den Jahren 2004 bis 2008 durchgeführte Studie "Lebensführungen und Geschlechterarrangements im Wandel. Aneignungspraktiken gesellschaftlicher Umbrüche am Beispiel von Beschäftigten im (ostdeutschen) Einzelhandel“. Insgesamt umfasst die Studie ein Sample von 25 Einzelhandelsbeschäftigen (neun Männer und 16 Frauen). Die im Sommer 2004 und im Winter 2005/2006 Befragten sind überwiegend ostdeutscher Herkunft, sie gehören unterschiedlichen Altersgruppen ( 21 bis 60 Jahre) an und leben in sehr unterschiedlichen Familien- und Lebensformen. Es wurden Mitarbeiterinnen und Mitarbeiter in Vollzeit-, Teilzeit- oder geringfügigen Beschäftigungsverhältnissen befragt. Die betrieblichen und qualifikatorischen Positionen erstrecken sich von der angelernten Aushilfe bis zur Filialleitung. Mit vier Befragten, so auch mit den hier vorgestellten, wurden nach eineinhalb Jahren ausführliche biografisch angelegte Wiederholungsinterviews geführt. Die Auswertung des Interviewmaterials erfolgte über vergleichende Einzelfallanalysen und über kleinschrittige Sequenzanalysen in heterogen zusammengesetzten Auswertungsgruppen. Einige anders gelagerte Ergebnisse der Studie sind bereits veröffentlicht worden (vgl. Völker 2010, 2008 2007, 2006). 
(2) Es zeigt sich bei den unterschiedlichen Indienstnahmen von Geschlecht bzw. Männlichkeit und den differenten Wahrnehmungen prekarisierter Arbeit, dass sich die unterschiedlichen praktischen Stellungnahmen der Interviewten nicht allein mit Rückgriff auf soziologische Wissensformen verstehen lassen. Was soziale Prozesse verstehbar und erklärbar macht, ist mitunter gar nicht mit sozialen Kategorien, d.h. mit Dimensionen, die sich allein auf gesellschaftliche Regeln und Dynamiken beziehen, zu beschreiben. Erforderlich ist vielmehr der Blick auf die Gebundenheiten und Bewegungen in verschiedenen sozialen, physischen oder geografischen Räumen und mit Bezug auf die Verknüpfungen von sehr unterschiedlichen Realitäten (etwa von biografischen Erfahrungen oder psychischen Konstellationen). Die Annäherung an die komplexen Lebensrealitäten der Einzelnen und die je spezifische Historizität ihrer Erfahrungen reicht dabei über eine disziplinäre Perspektive hinaus, weil sich die Artikulationen sozialer Praxis und prekärer Existenzweisen auf Phänomene beziehen, die nicht allein soziologisch (be-) greifbar sind.

Ein Beispiel: Für prekäre Leben in Erwerbslosigkeit können die Krise der Anerkennung, die Infragestellung der Lebbarkeit des eigenen Lebens und der Geschlechtsidentität von zentraler Bedeutung sein. Es können so Leidenserfahrungen durch die Friktionen zwischen Männlichkeit und Erwerbsarbeit (als soziologische Analysekategorien und normativ wirksame Selbstzuschreibungen) als bekannte Antworten auf die gleichermaßen soziologisch bekannten Herausforderungen einer postfordistischen Arbeitsgesellschaft partiell erklärt werden.

Leben in Erwerbslosigkeit bringen aber zugleich veränderte Dynamiken und Interferenzen von Zeit, Raum, Körper, Landschaft etc. hervor - etwa durch andere Zeitbezüge oder Bewegungsabläufe im physischen Raum. Sie produzieren Verknüpfungen und Praxen, die nicht allein mit den soziologischen Kategorien von Erwerbsarbeit, Männlichkeiten und sozialer Anerkennung zu begreifen sind, sondern unbekannte Antworten auf mitunter ungestellte Fragen nach veränderter Sozialität und Relationalität geben und damit zugleich die soziologischen Kategorien mitgestalten und verändern.

Im Sinne dieser methodologischen Berücksichtigung der Ungewissheit der
Umbruchprozesse und der Überlappungen und gegenseitigen Durchdringung von unterschiedlichen Wissensformen und Dynamiken, wurde gerade das Partikulare, die Komplexität des Einzelfalls rekonstruiert, um soziale Prozesse (be-)greifbarer zu machen. Ausgehend von den Analysen zeigten sich drei unterschiedliche Praxismuster. Mit diesen „Praktiken der Instabilität" (vgl. Völker 2006) wird nicht der Anspruch auf vollständige Repräsentanz des sozial Möglichen erhoben, dennoch geben die drei Modalitäten Hinweise auf markante Varianten sozialen Handelns in prekarisierten Arbeits- und Lebensverhältnissen. Die aufgefundenen Handlungsmodi lassen sich systematisch entlang von drei Dimensionen vergleichend beschreiben:

- der jeweiligen Aufladung von Geschlechtsidentitäten - also was sind „Frauen“ und „Männer“ bzw. was sollen sie tun?, - der Formen sozialer Einbindung und Lebensführungen und

- dem Umgehen mit Zukunftsunsicherheit.

Im Folgenden gehe ich den unterschiedlichen Praxisvarianten, Identifikationen und Selbstverortungen der befragten Männer nach. ${ }^{3}$ Beim dritten Praxismuster arbeite ich am Beispiel des Herrn V., seiner Biografie und der familialen Dynamik etwas breiter heraus, wie die ausgesprochen interessante Wahrnehmung der Prekarisierung von Arbeit und Leben als Chance der sozialen Situierung zustande kommt und was diese zunächst individuelle Konstellation dann in der Perspektive der Männlichkeits- und Milieuforschung soziologisch bedeuten kann.

\section{Praxisform I: Orthodoxe
Klassifizierungen}

In der Deutungs- und Handlungslogik der ersten Praxisvariante, die ich als „orthodoxe Klassifizierung "bezeichnet habe, markiert die Geschlechterdifferenz ein sozial höchst relevantes Ordnungsprinzip. Die Männer greifen auf die Binarität von Männlichkeit und Weiblichkeit zurück, um die eigene Erwerbsposition und ihre Vorstellungen der geschlechtlichen Arbeitsteilung mit Sinn auszustatten. Dabei dienen geschlechterdifferenzierende und hierarchisierende Klas- sifikationen dazu, verwundbare Lebensarrangements zu (re-)stabilisieren und soziale Forderungen in einer (naturalisierten) Geschlechterordnung zu verankern.

Für Herrn K. geht es mit seinen vergeschlechtlichten Vorstellungen zur Erwerbsarbeit (ernsthafte Männerarbeit und Familienernährerfunktion versus Frauenarbeit als minderwertige Beschäftigung) um die Artikulation der eigenen, männlichen Ansprüche an eine erfüllende Erwerbsarbeit, die die Gesellschaft ihm gegenwärtig verwehrt. Der 21-Jährige lebt allein und arbeitet zum Interviewzeitpunkt zur Aushilfe in einer Spielwarenkette. Seine Herkunftsfamilie stammt aus Polen, der Katholizismus ist im eigenen Alltag gegenwärtig und bestimmt die Vorstellungen über eine künftige Familie. Respektabilität ist für ihn mit dem „Besitz“ von Familie und einem vollwertigen Arbeitsplatz verknüpft. Er gibt seine vergleichsweise stabile Tätigkeit im Einzelhandel als gelernter Verkäufer für die freiberufliche Tätigkeit eines Tennislehrers auf.

Deutlich formuliert er seine Geringschätzung der Verkaufstätigkeit, er fühlt sich dort in seiner Männlichkeit zur Disposition gestellt:

„Nach den drei Jahren hab ich auch gemerkt, Einzelhandel is-für mich, persönlich, nichts. Also dies können Frauen machen, auch Männer natürlich, aber für`n Mann an ner Kasse sitzen iss, also is nich mein Leben, kann ich mir nicht vorstellen (...). Wenn ich im Einzelhandel arbeite, kann ich auch keine Frau und Kinder haben. Das ist

3 Ich fokussiere hier auf die Verknüpfung oder Entflechtung von Arbeit und Männlichkeit aus Sicht der befragten Männer. Alle Praxisvarianten, die hier an jeweils einzelnen Fallbeispielen dargestellt werden, haben sich auch bei weiteren befragten Männern und Frauen rekonstruieren lassen. Es handelt sich bei diesen Varianten sozialen Handelns keineswegs um nach Geschlecht differenzierte, also etwa um spezifisch ,männliche‘ Praktiken. Gleichwohl unterscheiden sich mitunter die Arten und Weisen, mit denen Frauen und Männer ihre jeweiligen Geschlechterverortungen mit diesen Handlungspraxen verknüpfen. So stellen sich jene Frauen, die sich orthodoxer Klassifizierungen bedienen, sehr offensiv als weibliche Zuverdienerinnen und "Hobbyarbeitnehmerinnen“ dar. Sie scheinen damit zunächst weit weniger in ihrer Geschlechtsidentität bedroht zu sein als die hierzu komplementäre männliche Position, deren Familienernährerfunktion erheblich unter Druck steht. Dass aber diese Version von Weiblichkeit und weiblicher Zuarbeit dann unter Wandlungszwang gerät, wenn das männliche Pendant des Familienernährers durch Arbeitsplatzverlust nicht mehr zur Verfügung ist, ist bereits jetzt absehbar. 
nen guter Beruffür ne Frau, die nen Mann hat, der Mann arbeitet wo anders, verdient gutes Geld und die Frau arbeitet hier und verdient eben auch nen bisschen was." 4

Solange Herr K. in der Spielwarenbranche überdauern muss, betreibt er zur notdürftigen Wahrung seiner männlichen Position tätigkeitsbezogene „boundary work“ (Heintz et al. 1997): Er verweigert die für ihn weiblich konnotierte Kassierertätigkeit und ist vorzugsweise für den Verkauf von Fahrrädern und Carrera-Autobahnen zuständig. Sozialen Sinn macht die starke Betonung von geschlechterdifferenzierenden Erwerbs- und Lebensarrangements, wenn es darum geht, dem empfundenen männlichen Anrecht auf ein Normalarbeitsverhältnis Plausibilität zu verleihen und entsprechend prekäre Arbeit für Männer zu skandalisieren, während sie für Frauen als Zuverdienerinnenmodell bei voller Verantwortung für die Familienarbeit akzeptiert wird. Insofern können die Praktiken von Herrn K. als Anknüpfung an fordistisch geprägte Erwerbsarbeits-, Geschlechter- und Männlichkeitsvorstellungen gelesen werden. Zugleich ist jedoch präsent, dass diese Vorstellungen bedroht und fragil sind.

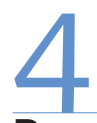

\section{Praxisform II: Erschöpfun- gen - Krise der (männ- lichen) Erwerbsarbeit}

Als zweite, die alltäglichen Praktiken bestimmende Erfahrung lässt sich die „Erschöpfung von zuvor selbstverständlichen institutionellen Arrangements", Integrationen und Teilhabechancen beschreiben. Hier sind für die befragten Männer Bezugnahmen auf geschlechterdifferenzierende Aufgaben und Ansprüche nicht unbedeutend, aber der Alltag und die Zukunft werden gerade in Nichtübereinstimmung mit diesen Leitvorstellungen erlebt und antizipiert. Insofern erschöpfen sich die fordistisch geprägten Vorstellungen und müssen umgearbeitet werden, weil sie immer weniger Möglichkeiten des praktischen Anschließens im Alltag finden. So wird versucht, mit dem Verlust der Erwerbsposition und dem Auflösen der Verknüpfung von Männlichkeit, Väterlichkeit und Erwerbsarbeit zu rechnen und sich in der Lebensführung für das Umgehen mit instabilen Situationen zu konditionieren.
Der 32-Jährige Herr D., ein gelernter Einzelhandelskaufmann, hat die Erfahrung gemacht, trotz aller Bildungsanstrengungen nicht verlässlich seine männlich/ väterliche Funktion in der Familienarchitektur einnehmen zu können. Seine Zeit in der Arbeitslosigkeit erinnert er als tiefe Identitätskrise. Es ist die Strukturlosigkeit der leeren Tage, vor allem aber der Verlust seiner Familienernährerposition und die Scham vor den Kindern, seiner Funktion als väterliches Vorbild nicht mehr gerecht werden zu können, die die Erfahrung der Erwerbslosigkeit bis in die Gegenwart handlungsrelevant sein lässt: „Man lebt in Tach hinein und dit is natürlich nich, wat unbedingt erfüllend ist. (...) Gerade wenn man so ne Familie hat, (...) is man ja stetig druff bedacht, halt irgendwo nen gewissen Tagesablauf drinne zu haben, um auch ne Vorbildfunktion für seine Kinder sein. Dis is erstmal janz janz wichtig. Steht zumindest an oberster Stelle. Und dann natürlich dit Geldliche. Das man och denn die Familie halten kann."

Herr D. ist heute bereits seit fünf Jahren Angestellter eines Telekommunikationsvermarkters in einem mittlerweile unbefristeten Beschäftigungsverhältnis, das er als einer der Letzten glücklich "abgefasst“ hat. Obgleich er sich relativ gut in seinem Unternehmen verankert wähnt - er ist zum stellvertretenden Filialleiter aufgestiegen -, empfindet er seine umsatzabhängige Bezahlung als tägliche Unsicherheit und Herausforderung, genug Geld zu verdienen um sich (s)eine Familie „leisten“ zu können. Deshalb geht es ihm und seiner Partnerin darum, sich beide mit vollem Einsatz und zielstrebig im Erwerbsbereich zu verankern und so dafür gewappnet zu sein, dass jederzeit eine der beiden Stellen „wegbrechen“ kann. Dies bedeutet auch, dass die jeweilige Tagesverantwortung für die Kinder innerhalb der Partnerschaft wechselt und ausgewogen über die Wochentage verteilt ist. Die Erfahrung des Arbeitsplatzverlusts motiviert hier eine Strategie des Einkalkulierens von Instabilität und Verwerfungen, die das Festhalten an Vorstellungen von geschlechtsdifferenzierenden Arbeitsteilungen untragbar und zu einer unakzeptablen Gefährdung des Lebenszusammenhangs macht (vgl. zu veränderten Geschlechterarrangements als Ressource zur Bewältigung von Prekarisierung den Beitrag von Klenner et al. in diesem Heft).
Praxisform III: Gelegen-
heitsorientierungen und
temporäre Arrangements

Als dritte Handlungsvariante hat sich gerade in der jüngeren Generation das „Setzen auf kurzfristige, spontane Gelegenheiten" gezeigt. Die Versuche zur sozialen Positionierung werden weniger in längerfristigen Laufbahnen gedacht als im mutigen Nutzen kontingenter Chancen. Wenn es darum geht, die Instabilität der Erwerbsverhältnisse und Ungewissheiten des sozialen Raums als Ressource zu deuten, dann engen zu straffe Geschlechterklassifikationen nur ein. Die Relevanz von Festlegungen über Männlichkeit und Weiblichkeit muss temporär immer wieder neu ausgelotet werden und sich dabei auch als sozial sinnvoll und Erfolg versprechend erweisen.

Auffällig ist in dem qualitativen Sample, dass diese Gelegenheitsorientierung besonders mit Entwurzelungserfahrungen und mit spezifischen Milieuzugehörigkeiten verbunden ist. Es handelt sich dabei um eher marginalisierte Gruppen, die bereits in historischer Perspektive nicht in das dominante Zeit-, Zukunfts- und methodische Lebensführungsregime der respektablen Arbeitnehmerinnen- und Arbeitnehmermilieus integriert waren (vgl. Vester et al. 2001, S. 26ff.). Die Einlagerung dieser Erfahrungen der Instabilität und Diskontinuität in aktuelle Umstellungsstrategien kann marginalisierten und entwurzelten Milieus durchaus zu Ressourcen im Umgehen mit Prekarisierung werden. Bewegungen des sozialen Schwebens oder Driftens sind oftmals in der Elterngeneration der von mir Befragten angelegt - z.B. als sozial marginalisierte, von staatlichen Übergriffen geprägte Geschichte der Elternfamilie und der eigenen Kindheit wie dem folgenden Beispiel von Herrn V.. Die Unbestimmtheiten eines nicht eindeutig strukturierten, ja löchrigen gesellschaft-

\footnotetext{
4 Die Interview-Zitate sind in der gesprochenen Sprache direkter Interaktion, die durch Satzabbrüche, Pausen, Betonungen, Wiederholungen gekennzeichnet ist, wiedergegeben. Die Zitate sind in dem regionalen Dialekt belassen, da jede "Übersetzung" einen neuen Text produziert hätte, es an dieser Stelle aber darum geht, die Ausführungen der Befragten zu spezifischen Aspekten möglichst nah an ihren Deutungen zu dokumentieren.
} 
lichen Raums sind dann weniger eine Bedrohung einer etablierten anerkannten Position und des entsprechenden Selbstverständnisses, sondern sie werden als Chance erfahren. Hier, in der Ungewissheit der entsicherten Arbeitsverhältnisse, ist mehr möglich als in einer fest gerahmten, marginalisierten Randlage der vergangenen, hoch integrierten fordistischen Arbeitsgesellschaft. Im unbestimmten Raum steigt aus Sicht des Befragten die Chance, sich (mit enormem Kraftaufwand) gegen die weniger dichte und verfestigte soziale Schwerkraft am eigenen Schopf hochziehen zu können und sich selbst zu erfinden. Dies kann dann auch bedeuten, dass die Geschlechterdifferenz eine weniger stark markierte, sondern situativ produzierte oder eben vernachlässigte Ordnungskategorie wird.

\section{1 "CHANCEN" DER PREKARITÄT}

Herr V. ist zum Zeitpunkt des Erstinterviews im Jahr 200635 Jahre alt und seit einem guten Jahr „Verkaufsoptimierer" für einen Haushaltswarenanbieter in dem hochwertigen Qualitätssegment der Branche. Sein Shop ist Bestandteil der größeren Haushaltswarenabteilung eines Kaufhauses; neben ihm gibt es zwei weitere Verkaufskräfte, die für externe Haushaltswarenanbieter mit einem etwas anderen Sortiment ihr Geschäft in unmittelbarer Nähe betreiben. Herr V. arbeitet gänzlich auf Provisionsbasis, ist selbstständig und alleinverantwortlich für seinen Gewinn.

In seiner diskontinuierlichen Berufslaufbahn ist die aktuelle Tätigkeit sowohl hinsichtlich des Status als auch der Verdienstmöglichkeiten vorläufiger Höhepunkt eines Wegs immer neuer SelbstErfindungen. 1971 in der DDR geboren, beendet er kurz vor dem Systemumbruch seine Ausbildung zum Bekleidungsfacharbeiter. Seine Anstellung in der industriellen Akkordfertigung von Bekleidung kündigt er nach der Wende, da ihm die Arbeit zu monoton ist. Anschließend „überwintert" er fünf Jahre als angelernter Arbeiter in dem Lager eines Kaufhauses. Nach dem Ende seiner einzigen langjährigen Partnerschaft strebt er nach einer anspruchsvolleren Position. Als ein „Kumpel“ seinen Job als externer Mitarbeiter im High-TechSpielwarenbereich verliert, kann Herr V. ihn „beerben“. Wie wenig rechtlich abgesichert und prekär diese selbstständige Position des Verkaufsoptimierers ist, zeigt ihr abruptes Ende acht Jahre später. Seine Auftraggeber kündigen den Vertrag von einem Tag auf den anderen. Es folgt ein Jahr Arbeitslosigkeit, die Situation ist ökonomisch höchst prekär: Als ehemals Selbstständiger bekommt er kein Arbeitslosengeld und zehrt sein Erspartes auf. Er ist bereits auf der Suche nach einer preisgünstigeren Wohnung, als er einmal mehr über einen „Kumpel“ das Angebot bekommt, als Verkaufsoptimierer für eine Haushaltswarenmarke tätig zu werden. Herr V. begrüßt die Selbstständigkeit dieser Position ausdrücklich.

Nachvollziehbar wird diese riskante Orientierung auf eine Erwerbstätigkeit, die ganz individuell den Erfolg (und nicht die Anstrengung) des eigenen Handelns belohnt, wenn man die Bedeutung der Erwerbsarbeit für die eigene Selbst(emp-) findung innerhalb der biografischen und psychischen Dynamik rekonstruiert.

\subsection{ERWERBSARBEIT - SICH VERTIE- FEN UND ERLEBEN}

Die Verkaufstätigkeit ist für Herrn V. der zentrale Dreh- und Angelpunkt seines Lebens. Er "geht in die Tiefe", versetzt sich in die Wünsche der Kundschaft und forscht nach optimalen Lösungen. Hier, im Kontakt, spürt er sich als soziales Wesen, sein Können, seine (Überzeugungs-)Kraft. Seit nunmehr über zehn Jahren ohne eine (von ihm jedoch sehr gewünschte) Partnerschaft lebend, organisiert sich Herr V. auf der selbst geschaffenen Bühne seines Verkaufsbereichs schöne Erlebnisse und stellt sich der Kundschaft mit all seinen Ideen zur Verfügung:

„Diese Freude eigentlich entgegen zu bekommen, dieses Glückliche (...). Die Stammkunden wiederzusehen und zu sagen: "Mensch, geil, hat mir jefallen" oder dass man sagt „Mensch, pass ma uff, da kriegen wa ne Lösung hin, dit sollte doch keen Problem sein", also den Kunden nicht verliert und wieder neu ufffängt. Also dieser Kreislauf, jemand immer um sich rum zuhaben. (...) Is natürlich noch ne andere Sache, wenn man janz alleene is, also jetzt och keen Partner hat und denn is der fast nen Ersatz vom Partner."

Für ein derartiges Hineinbegeben in den Arbeitsbereich mit Haut und Haaren, Leib und Seele, finden sich Erklärungen in der biografischen Konstellation. Im Bereich der sozialen Nahbeziehungen hat Herr V. die schmerzhafte Erfahrung gemacht, auf sich selbst, auf sein ganz eigenes
Vermögen der Verankerung und Stabilisierung angewiesen zu sein.

\subsection{ERFAHRENER MANGEL - WAS IST "ZUHAUSE"?}

In seiner Kindheit muss Herr V. erleben, dass auf die von unterschiedlichen Seiten erklärten Zusicherungen, sich um ihn zu kümmern, nur sehr begrenzt und temporär Verlass ist. Seine Mutter ist Angestellte bei der Post und alleinerziehend. Sie ist in ihrem Leben nicht glücklich und bringt sich und den Sohn nur mit Mühe durch. Zur Zeit der Einschulung des Sohnes arbeitet sie in Nachtschicht und schläft tagsüber, für den Sohn ist sie kaum ansprechbar. Am Tag ist dieser häufig bei den Eltern eines Schulfreundes. Nachts allerdings, wenn die Mutter arbeitet, weint er und zieht damit die Aufmerksamkeit der Umwelt auf sich. Die Behörden werden von besorgten Nachbarn eingeschaltet, der Mutter wird die Vernachlässigung des Sohnes vorgeworfen und das sechsjährige Kind gerät so in die Mühlen des erzieherisch eingreifenden Staatsapparates - die kommenden viereinhalb Jahre muss er in einem Erziehungsheim verbringen. Letztlich begleiten zwei Erfahrungen Herrn V. seit seinen Kindheitstagen: Er erlebt temporäre Unterstützungen, die jedoch nicht dauerhaft tragfähig sind und sich auch gegen ihn wenden können. Eine geborgene und sichere Eingebundenheit gibt es nicht, sondern-wie er es nennt - „,breaks“, Brüche, Abschiede, die sich daraus ergeben, dass die Unterstützungen von zugewandten Nachbarn, einer Lehrerin, einer Kabarettgruppe oder auch die Anstrengungen der Mutter immer wieder überfordert und deshalb begrenzt sind. Herr V. fällt also nicht durchs Netz, aber - das ist die zweite Erfahrung - aufgehoben ist er nicht, sondern im Bereich der persönlichen Nahbeziehungen beschädigt.

Diese Verletzungen setzen sich aus seiner Perspektive auch in seiner Partnerschaftsgeschichte fort: Hier wird er zwei Mal „fallen gelassen" und erholt sich nach eigenem Bekunden von diesen Erfahrungen nur schwer. Seit über zehn Jahren lebt er nicht nur ohne Partnerschaft, sondern auch ohne etwas, was er als Zuhause bezeichnen könnte. Sein Problem, eine Wohnung überhaupt bewohnbar zu machen und dort länger als ein paar Monate zwischen Kartons zu verweilen, ist ein Beispiel dafür, dass hier ein Vermögen von ihm ab- 
verlangt wird, das er selbst - nach eigenem Bekunden bis auf die fünf Jahre gemeinsamen Lebens mit der Freundin - nicht durch andere erfahren hat. Das ermangelte Zuhause als einem wohnlich-gestalteten Raum des Aufgehobenseins identifiziert er mit Frauen, mit einem abstrakt bleibenden Weiblich-Mütterlichen. Gegenwärtig führt er ein Nomadendasein, dessen gedanklicher Fluchtpunkt die Sesshaftigkeit mittels der Gründung einer Familie ist. Diejenige, die ihm helfen soll, ein Zuhause aufzubauen, ist „die richtige Frau“, denn er selbst weiß nicht, wie das gehen soll. Er resümiert: „Also ick bin immer am rumtengeln und leb irgendwie so mein Leben, aber zuhause - nicht. Und dit is eigentlich mein Schwachpunkt, den ick jetzt für mich rauskristallisiere."

\subsection{PREKÄRES LEBEN - EIN INDIVI- DUELLER DRAHTSEILAKT}

Wenn ich den Pfad der biografischen Konstellation verlasse und die skizzierten Erfahrungen auf die Präferenz für das Arbeitsverhältnis als selbstständiger Verkaufsoptimierer beziehe, dann ergibt dieses für Herrn V. sehr viel Sinn. Da ist einmal die ausgesprochen negative Erfahrung mit institutionell reguliertem Handeln, bei dem er selbst in seinem Handeln und Wollen völlig außer Acht gelassen wird. Gleichzeitig macht er auch die Erfahrung, dass er auf jene, die sich für ihn einsetzen, nur begrenzt bauen kann. Den verlässlichen Boden unter seinen Füßen muss er aus sich selbst schöpfen und selbst schaffen. Dafür bedarf er eines angemessenen Entfaltungsraums. Dieser Raum ist für ihn aufgrund seiner Herkunft aus einem randständigen prekären Milieu nicht so ohne Weiteres vorgesehen. Er verfügt weder über einen Ort familiärer sozialer Bindung noch über eine gefestigte Position im sozialen Raum. Es geht also für ihn darum, sich selbst und seine Position in einem ungewissen gesellschaftlichen Raum zu erfinden, darzustellen und sich auf diese Weise zu verankern - das sieht er als ,Chance' der Prekarität.

\section{Entsicherte Geschlech- terordnung: verschobene Klassifikationen und die Krise der Reproduktion}

Die drei vorgestellten Varianten sozialer Praxis geben Hinweise darauf, wie unterschiedlich starr und mehr oder weniger differenzsetzend die Vorstellungen von Männlichkeit und Weiblichkeit der Befragten im Hinblick auf ihre Erwerbsposition sind und inwieweit diese Vorstellungen mit den (unbewussten) Strategien der sozialen Reproduktion verknüpft werden. Es wird dabei allerdings nicht nur die Erwerbsarbeit als umkämpftes Feld sichtbar, sondern auch die große Gefährdung und Krise des Bereichs der physischen Reproduktion, der Selbstsorge und Sorge um andere. Mit Blick auf die eingangs dargestellte Diskussion in der Männlichkeitsforschung verweisen die Befunde auf die Notwendigkeit, die gerade nach sozialen Milieus sehr heterogenen Erfahrungen, Kompetenzen und Befunde mit einzubeziehen. Diese bestätigen die Vermutung einer überwiegenden Retraditionalisierung in den unteren Zonen des gesellschaftlichen Raums nicht, im Gegenteil. Es zeigt sich bei den Strategien von jungen Männern (und auch von jungen Frauen, wie weitere Fallanalysen gezeigt haben, vgl. Völker 2010) eine Homologie zwischen der Unbestimmtheit der sozialen Lage eines negativ privilegierten Milieus und einer wachsenden praktischen Unbestimmtheit der Geschlechterrollen, die sich für die Handelnden als ertragreich und Erfolg versprechend für ihre soziale Positionierung erweist. Insofern haben wir es eben nicht allein mit dominierten Männlichkeiten zu tun, die orthodoxe Geschlechterklassifikationen reaktualisieren, sondern mindestens ebenso mit innovativen Strategien der Selbstpositionierung, die im Bereich der Erwerbsarbeit Geschlecht als Ordnungskategorie entdramatisieren und in den Hintergrund treten lassen. Es gelingt einigen Akteuren und Akteurinnen offenbar, aus der sozialpositionellen und geschlechterdifferenzierenden Unsicher- heit oder partiellen Offenheit Handlungsfähigkeit zu schöpfen und sich mit hohem sozialem und physischem Kraftaufwand zu erfinden und sozial zu verankern. Dies bedeutet aber nicht, dass sie trotz aller Handlungsfähigkeit über einen Raum der sozialen und physischen Reproduktion wirklich verfügen. Wir haben es mit einer komplexen Konstellation der Entsicherung von sozialen und geschlechterdifferenzierenden Integrationen und Begrenzungen im Erwerbsbereich und einer Ernennung und Verdrängung des Ortes der Regeneration und Reproduktion zu tun.

Dies erklärt in den hier individuell biografisch rekonstruierten Strategien sozialen Handelns eine in den letzten beiden Praxisvarianten relative Offenheit bezüglich der Klassifikationen der Geschlechterdifferenzierung im Erwerbsbereich und eine Ratlosigkeit gegenüber der privaten und generativen Reproduktion. Dies stützt den Befund der für die postfordistische Gegenwart aktuell konstatierten „Krise der Reproduktion“ (Aulenbacher 2009; vgl. auch Jürgens in diesem Heft). In der individuellen Bearbeitung wird diese Krise sehr unterschiedlich artikuliert: In den ersten zwei Varianten geht es um Eindämmung der Unsicherheit durch die Reformulierung von getrennten Frauenund Männerwelten (orthodoxe Klassifizierungen) oder durch die methodischkalkulierende Integration der Krise der männlichen Erwerbsarbeit mittels der partiellen Aufweichung von geschlechterdifferenzierenden Lebensführungen. Die dritte Variante sucht nicht Unsicherheit zu bannen, sondern aus ihr zu schöpfen. Sie findet aber für den Bereich der physischen Regeneration, der Zusammenlebens- und Beziehungsformen und der Generativität keine Lösung. Nicht nur in dem dargestellten Fallbeispiel wird dieser Mangel durch Weiblichkeitsphantasmen zu adressieren und zu „heilen“ versucht. Aus Sicht von politisch intervenierenden Akteurinnen und Akteuren ginge es daher darum, dieses Phantasmatische rückzuführen in eine Politik des Raum-Schaffens für reproduktive, sorgende und geschlechtlich unbestimmte Tätigkeiten, die erst ein gutes, umsorgtes und gehaltenes Leben ermöglichen. 
Aulenbacher, B. (2009): Arbeit, Geschlecht und soziale Ungleichheiten Perspektiven auf die Krise der Reproduktion und den Wandel von Herrschaft in der postfordistischen Arbeitsgesellschaft, in: Arbeits- und Industriesoziologische Studien 2 (2), S. 61-78

Bourdieu, P. (1998): Prekarität ist überall, in: Bourdieu, P.: Gegenfeuer, Konstanz, S. 96-102

Bude, H./Willisch, A. (Hrsg.) (2008): Exklusion. Die Debatte über die „Überflüssigen“, Frankfurt a. M.

Castel, R. (2000): Die Metamorphosen der sozialen Frage. Eine Chronik der Lohnarbeit, Konstanz

Castel, R./Dörre, K. (Hrsg.) (2009): Prekarität, Abstieg, Ausgrenzung. Die soziale Frage am Beginn des 21. Jahrhunderts, Frankfurt a.M./ New York, S. 381-386

Dörre, K. (2007): Prekarisierung und Geschlecht. Ein Versuch über unsichere Beschäftigung und männliche Herrschaft in nachfordistischen Arbeitsgesellschaften, in: Aulenbacher, B./Funder, M./Jacobsen, H./ Völker, S. (Hrsg.): Arbeit und Geschlecht im Umbruch der modernen Gesellschaft, Forschung im Dialog, Wiesbaden, S. 285-301

Egert, G./Hagen, H./Powalla, O./Trinkaus, S. (2010): Praktiken der Nichtmännlichkeit - Prekär-Werden Männlicher Herrschaft im ländlichen Brandenburg, in: Manske, A./Pühl, K. (Hrsg): Prekarisierung zwischen Anomie und Normalisierung. Geschlechtertheoretische Bestimmungen, Münster, S. 186-209

Götz, I./Lemberger, B. (Hrsg.) (2009): Prekär arbeiten, prekär leben. Kulturwissenschaftliche Perspektiven auf ein gesellschaftliches Phänomen, Frankfurt a. M.

Heintz, B./Nadai, E./Fischer, R./Ummel, H. (1997): Ungleich unter Gleichen. Studien zur geschlechtsspezifischen Segregation des Arbeitsmarktes, Frankfurt a. M.

Klenner, C./Klammer, U. (2010): Frauen sorgen fürs Geld - und die Familie, in: Böckler Impuls (11), S. 6-7

Krüger, H. (1995): Dominanzen im Geschlechterverhältnis: zur Institutionalisierung von Lebensverläufen, in: Axeli Knapp, G./ Becker-Schmidt, R. (Hrsg.): Das Geschlechterverhältnis als Gegenstand der Sozialwissenschaften, Frankfurt a. M./New York, 195-219

Lengersdorf, D./Meuser, M. (2010): Wandel von Arbeit - Wandel von Männlichkeiten, in: Österreichische Zeitschrift für Soziologie, Themenheft Arbeit: Alltag und Biografie im Wandel 35 (2), S. 89-103
Manske, A./Pühl, K. (Hrsg.) (2010): Prekarisierung zwischen Anomie und Normalisierung, Geschlechtertheoretische Bestimmungen, Münster Scholz, S. (2009): Männer und Männlichkeiten im Spannungsfeld zwischen Erwerbs- und Familienarbeit, in: Aulenbacher, B./Wetterer, A. (Hrsg): Arbeit. Perspektiven und Diagnosen der Geschlechterforschung, Münster, S. 82-99

Vester, M./von Oertzen, P./Geiling, H./Hermann, T./Müller, D. (2001): Soziale Milieus im gesellschaftlichen Strukturwandel. Zwischen Integration und Ausgrenzung, Frankfurt a. M.

Völker, S. (2006): Praktiken der Instabilität. Eine empirische Untersuchung zu Prekarisierungsprozessen, in: Aulenbacher, B./Bereswill, M./ Löw, M./Meuser, M./Mordt, G./Schäfer, R./Scholz, S. (Hrsg.): FrauenMännerGeschlechterforschung, State of the Art, Münster, S. 140-154 Völker, S. (2007): Prekäre Transformationen - herausgeforderte Lebensführungen, in: Bock, U./Dölling, I./Krais, B. (Hrsg.): Prekäre Transformationen. Pierre Bourdieus Soziologie der Praxis und ihre Herausforderungen für die Frauen- und Geschlechterforschung, Querelles-Jahrbuch für Frauen- und Geschlechterforschung, Göttingen, S. 176-194 Völker, S. (2008): Entsicherte Verhältnisse - (Un)Möglichkeiten fürsorglicher Praxis, in: Berliner Journal für Soziologie, Schwerpunktheft "Care - Black Box der Arbeitspolitik" 18 (2), S. 282-306

Völker, S. (2010): "Nem Kind nun vorzumachen, du kriegst 'ne Lehrstelle; ist für mich unrealistisch". Biografische Konstellationen in prekarisierten Erwerbsarbeitsverhältnissen - ein Fallbeispiel, in: Österreichische Zeitschrift für Soziologie, Themenheft Arbeit, Alltag und Biografie im Wandel (2), S. 38-53

Wagner, A./Wiethold, F. (2009): Prekäre Beschäftigung und Geschlecht, in: Kurz-Scherf, I./Lepperhoff, J./Scheele, A. (Hrsg.): Feminismus: Kritik und Intervention, Münster, S. 197-213

Wedgwood, N./Connell, R.W. (2008): Männlichkeitsforschung: Männer und Männlichkeiten im internationalen Forschungskontext, in: Becker, R./Kortendiek, B. (Hrsg.): Handbuch Frauen- und Geschlechterforschung, Theorie, Methoden, Empirie, Wiesbaden, S. $116-125$

Young, B. (1998): Genderregime und Staat in der globalen NetzwerkÖkonomie, in: Prokla 111, 28 (2), S. 175-198 